\title{
A simple method to facilitate oral surgery in patients with orthodontic appliances
}

\author{
Sir, \\ Mopping with gauze during surgery is essential to \\ keep the field bloodless and improve visualisation of \\ underlying structures. In patients with cleft lip and palate \\ who are undergoing orthodontic treatment, performing a \\ secondary corrective procedure on the lip or a procedure \\ for closure of nasolabial fistula becomes cumbersome as \\ the gauze piece, which is used for mopping, repeatedly \\ gets stuck in the orthodontic clips [Figure 1]. \\ We report a simple technique to prevent the gauze \\ getting stuck in the orthodontic clips. An aluminium foil
}




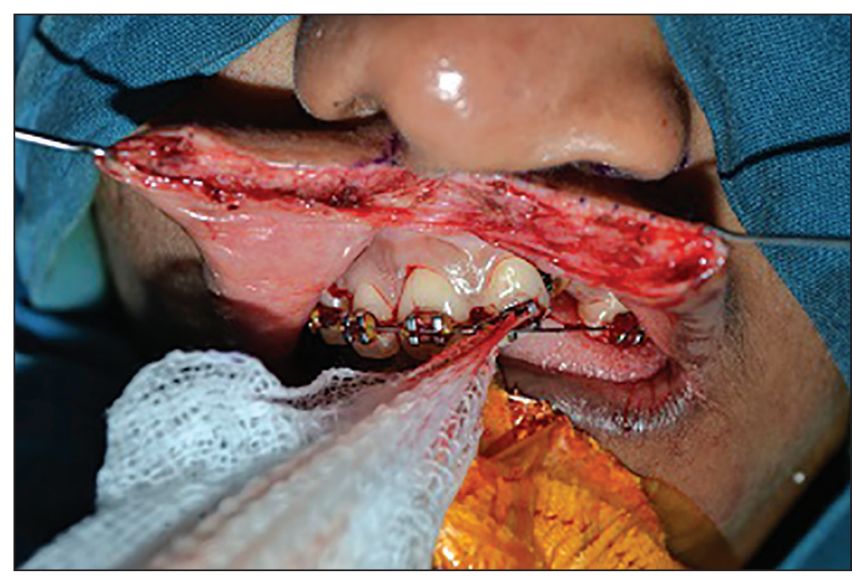

Figure 1: Picture depicting gauze piece stuck with orthodontic clip

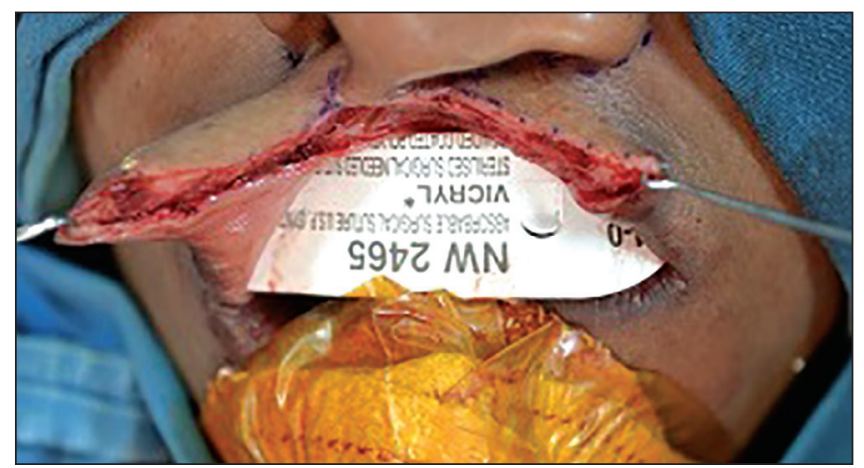

Figure 2: Picture depicting placement of suture foil in gingivolabial sulcus or the hard paper of the suture pack could be placed in the gingivolabial sulcus, covering the orthodontic clips, which avoids direct contact of the gauze with the clip [Figure 2]. This simple measure makes the surgeon very comfortable during procedure and saves a lot of time.

We searched the literature and to the best of our knowledge, could not find any measure like this, which helps the surgeon during procedure.

\section{Janarthanan Ramu, Abhijeet Wakure, Mohit Sharma}

Department of Plastic Surgery, Amrita School of Medicine, Amrita Vishwa Vidyapeetham University, Kochi, Kerala, India

Address for correspondence: Dr. R. Janarthanan, Department of Plastic Surgery Amrita School of Medicine, Kochi - 682 041, Kerala, India.

E-mail: ramjana98@yahoo.co.in

\begin{tabular}{|l|l|}
\multicolumn{3}{|c|}{ Access this article online } \\
\hline Quick Response Code: & Website: \\
\hline
\end{tabular}

